\title{
EEG signal classification for drowsiness detection using wavelet transform and support vector machine
}

\author{
Novie Theresia Br. Pasaribu, Timotius Halim, Ratnadewi, Agus Prijono \\ Department of Electrical Engineering, Universitas Kristen Maranatha, Indonesia
}

\begin{tabular}{|c|c|}
\hline Article Info & ABSTRACT \\
\hline Article history: & \multirow{9}{*}{$\begin{array}{l}\text { There are several categories to detect and measure driver drowsiness such as } \\
\text { physiological methods, subjective methods and behavioral methods. The } \\
\text { most objective method for drowsiness detection is the physiological method. } \\
\text { One of the physiological methods used is an electroencephalogram (EEG). In } \\
\text { this research wavelet transform is used as a feature extraction and using } \\
\text { support vector machine (SVM) as a classifier. We proposed an experiment of } \\
\text { retrieval data which is designed by using modified-EAR and EEG signal. } \\
\text { From the SVM training process, with the 5-fold cross validation, Quadratic } \\
\text { kernel has the highest accuracy } 84.5 \% \text { then others. In testing Driving- } 2 \\
\text { process } 7 \text { respondents were detected as drowsiness class, and } 3 \text { respondents } \\
\text { were detected as awake class. In the testing of Driving-3 process, } 6 \\
\text { respondents were detected as drowsiness class, and } 4 \text { respondents were } \\
\text { detected as awake class. }\end{array}$} \\
\hline Received Mar 18, 2020 & \\
\hline Revised Mar 17, 2021 & \\
\hline Accepted Apr 29, 2021 & \\
\hline Keywords: & \\
\hline Drowsiness & \\
\hline Electroencephalogram & \\
\hline Support vector machine & \\
\hline Wavelet transform & \\
\hline
\end{tabular}

This is an open access article under the $\underline{C C B Y-S A}$ license.

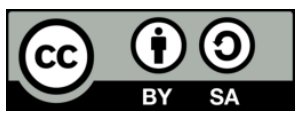

Corresponding Author:

Novie Theresia Br. Pasaribu

Department of Electrical Engineering

Universitas Kristen Maranatha

Jl. Surya Sumantri No.65, Bandung, Indonesia

Email: novie.theresia@eng.maranatha.edu

\section{INTRODUCTION}

The everyday activities of human life require transportation. Transportation is used to travel from one place to another. One of the transportation methods is done by car. Driving a car is an activity that requires high level of concentration, because driving for a long time can cause fatigue. Fatigue causes drowsiness which results in a decreased level of concentration in the driver which can result in a traffic accident. One effort that can be done to prevent traffic accidents due to drowsiness on the driver is by detecting drowsiness before the accident occurs.

There are several categories to detect and measure driver drowsiness such as physiological methods, subjective methods and behavioral methods [1]. There are several subjective methods, testing the respondent based on their subjective assestment such as karolinska sleepiness scale (KSS) and stanford sleepiness scale (SSS). Brown et al. [2] used KSS to observe the scale of sleepiness in nurses World Health Organization worked after shifts or when shifts were carried out, and the result was that nurses were detected to feel more drowsy when there was a shift in compared to after a shift. Jewett et al. [3], in the study of drowsiness detection with the SSS and psychomotor vigilance task (PVT), information is obtained by observing the slow- motion response in humans when drowsy with varying sleep times. In a drowsy condition, human response is slower than the awake condition. PVT has better performance than SSS performance. Some evidence used in the PVT test saw that a person who is drowsy usually has decreased eye pupil and slower motoric reaction speed. The PVT test observes the reaction of the respondent onlooking number on display. In our research [4] observed the performance of female and male drivers in PVT-based driving. Performance 
test from female and male driver have the same fatigue result, that female driver' $s$ collisions are higher than male driver' s collisions.

Drowsiness detection and measurement using behavioral methods, focus in facial expression such as: head and eye position/ state and frequent yawning [1]. Measure drowsiness using eye aspect ratio (EAR) is one of the behaviour methods. In the research by Mehta [5], a mild drowsiness detection system using Android was developed that can be used in real-time. The method used is image processing on recorded video frames, to detect faces using landmarks, then calculating the EAR and eye closure ratio (ECR) to detect driver sleepiness based on adaptive thresholding with a threshold value of 0.25 . If the EAR value is less than the threshold value, it indicates a state of fatigue. The random forest classifier is used as a classifier with an accuracy of $84 \%$. In our research proposed a modification of EAR, by using EAR threshold that calculating from EARmin dan EARmax design for individually driver [6].

The most objective method for drowsiness detection is the physiological method [1]. One of the physiological methods used is an electroencephalogram (EEG). EEG is done by recording human brain signals. There are several research analysing EEG signals for driver's drowsiness detection [7]-[16]. EEG signals can be extracted with a variety of methods such as wavelet transforms [17]-[18], fast fourier transform (FFT) [18], dan autoregressive model (AR) [19].

In the research by $\mathrm{Li}$ and Chung [18], design and development drowsiness detection system and combines with support vector machines (SVM). Hardware design using photoplethysmography (PPG) sensor and heart rate variability (HRV), verified based on the percentage of eyelid closure (PERCLOS) pupil. PPG signal and then classify driver as drowsy or alert. From this research classification using wavelet feature have higher accuracy than fast fourier transform (FFT). In Pauly research in drowsiness detection used a webcam camera that has a low resolution to support detect percentage of eyelid closure (PERCLOS), combining histogram of oriented gradients (HOG) and SVM. The final results from the system are compared with observations by eyes [20]. In Zhang research, designing drowsiness detection for high-speed trains based on drivers using wireless EEG, using FFT and the classification with SVM reaches 90.70\% [21].

Therefore in this research, we propose an experiment of data retrieval which is designed by using modified-EAR and EEG signal. In this research, using the neuroheadset emotiv EPOC to see the behaviour EEG signals to EAR's driver. EEG signal will classify by using SVM and wavelet transform as a feature extraction for drowsiness detection. The early drowsiness detection system is designed, so Alpha waves, Beta waves and Theta waves from EEG signal used in this research (start from the conditions's someone is still allertness, relax condition until the light sleep).

\section{THEORY AND DESIGN SYSTEM DROWSINESS DETECTION}

2.1. Theory

The following are theories used in the process of drowsiness detection based on EEG signals using SVM: EEG, wavelet transform and SVM.

\subsubsection{Electroencephalogram (EEG)}

Electroencephalogram (EEG) is an instrument to capture electrical activity in the brain. EEG signals are divided into six groups of brain waves that have different frequency ranges. The six main groups of EEG signals, namely [22]:

- Delta waves $(<4 \mathrm{~Hz})$, related to deep sleep.

- Theta waves $(4-8 \mathrm{~Hz})$, appears when someone is in a state of sleepiness and light sleep.

- Alpha waves $(8-13 \mathrm{~Hz})$, appears when someone is in a relaxed state and the strongest energy occurs in the frontal and occipital cortex.

- Mu wave (8-12 Hz), related to motor activity and can be used to recognize the purpose of one's movements.

- Beta waves (13-30 Hz), related to the level of alertness and concentration of a person.

- Gamma waves (30-50 Hz), are associated with one's mental activities such as creativity and problem solving.

\subsubsection{Wavelet transformation}

Wavelet transformation is the development of the fourier transformation so that it has the same way of working which is to break the signal into several parts. The difference is that the Fourier transform provides the frequency information of the signal but does not provide the time information, whereas the wavelet transform provides the time and frequency information of the signal. Wavelet transform is suitable to analyze non-stationary signals, different from fourier transform is not suitable for non-stationary signals [23]. 
Wavelets are a family of functions produced by the basis wavelets called mother wavelets [24]. The two main operations that underlie the wavelet are translation and scaling. Translation is a form of transformation of the time domain. Scaling is a form of transformation of frequency, with the scale value inversely proportional to the frequency value. Mathematically, the basic functions of wavelets are written as (1) $[24]:$

$$
\psi_{a, b}(t)=\frac{1}{\sqrt{|a|}} \psi\left(\frac{t-b}{a}\right)
$$

where $b=$ location parameter, $a=$ scaling parameter, $\mathrm{t}=$ time. Wavelet transform also consists of several types of families, namely Daubechies, Symlets, Coiflets, and others [24].

\subsubsection{Support vector machine (SVM)}

Support vector machine (SVM) was first introduced by Vapnik as a superior method in the field of pattern recognition [25]. SVM is a classifier based on a linear discriminant function, that can be used as a binary classifier [26]. The concept of SVM is trying to find the best hyperplane that functions as a separator of two classes in the input space. Hyperplane is a horizontal plane that functions as a class separator. The best separating hyperplane is obtained by finding the maximum margin. Margin is the distance of the hyperplane from the closest pattern commonly called a support vector [27]. There are two pattern (positive objects and negative objects) which will separate into two classes, Figure 1 shows some patterns that are members of two classes, namely -1 and +1 . The patterns incorporated in class -1 can be formulated as:

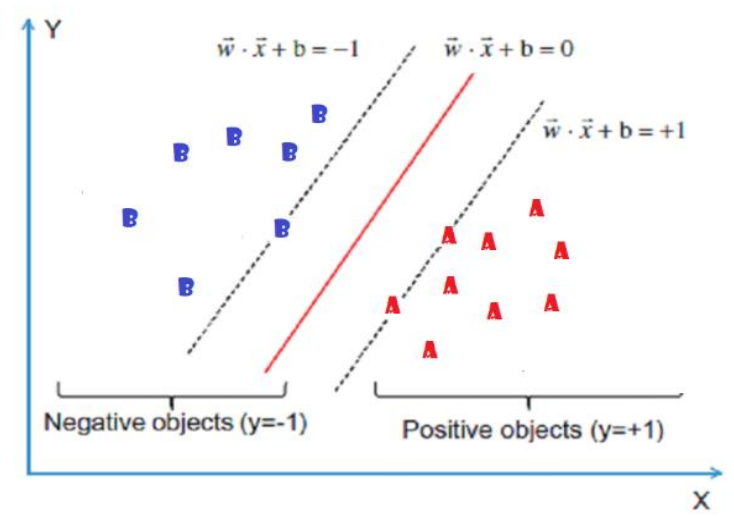

Figure 1. SVM hyperplane that separates the two classes [27]

$$
\vec{w} \cdot \vec{x}+b=-1
$$

While the patterns incorporated in +1 class can be formulated as (3).

$$
\vec{w} \cdot \overline{\vec{x}}+b=+\hat{1}
$$

To separate the two patterns perfectly it takes a hyperplane of dimension d which is formulated as (4).

$$
\vec{w} \cdot \vec{x}+b=0
$$

$\vec{x}_{i}:$ Input vector

$\vec{w}:$ Weight vector

$b:$ Bias

Cases that occur in the real world are rarely linearly separable; to overcome this problem the SVM was modified by including kernel functions. In non-linear SVM, the data $\vec{x}$ is first mapped by the function 
$\phi(x)$ to the higher dimensional vector space [23]. In this new vector space, the hyperplane that separates the two classes can only be constructed. This concept can be seen in Figure 2.

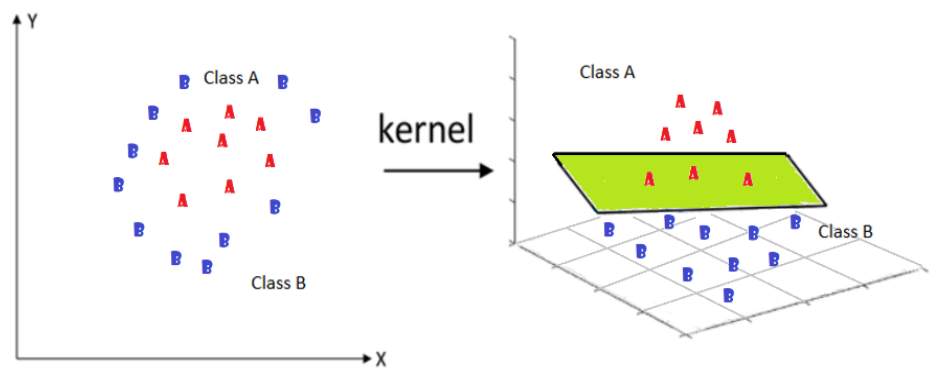

Figure 2. The idea of kernel trick SVM [27]

In Figure 2, it is shown that the data in the two dimensions input space cannot be linearly separated, and then the function $\phi$ maps each data to a higher dimension, so that the two classes can be separated linearly. Then the training process is the same as in the linear SVM. The optimization process in this phase requires the calculation of the dot product two examples in the new vector space. The dot products of the two vectors $\left(\mathrm{x}_{\mathrm{i}}\right)$ and $\left(\mathrm{x}_{\mathrm{j}}\right)$ are denoted as $\Phi\left(\mathrm{x}_{\mathrm{i}}\right)$. $\Phi\left(\mathrm{x}_{\mathrm{j}}\right)$. The dot product values of these two vectors can be calculated indirectly, that is, without knowing the transformation function $\Phi$. This computational technique is called Kernel Trick, which calculates the dot product of two vectors in a new vector space by using the components of both vectors in the original vector space. Several types of kernels in the SVM: linear kernel, polynnomial kernel and gaussian kernel [26].

\subsection{Design system drowsiness detection}

\subsubsection{Data collection scenarios}

Based on the accident traffic data from Korlantas Indonesia in 2018, there are three groups of accident victims namely at the age of 15-19 years old, 20-24 years old and 25-29 years old [15]. And also from the National Highway Traffic Safety Administration (NHTSA), crashes occurred during the period between 1989 and 1993 in the United States, there are approximately 100,000 crashes per year identified with drowsiness, the highest road traffic death by age range are 15-29 [1].Therefore in this experiment, the ages range of respondent are 19-26 years old, with a total of ten respondent in good health and have sufficient sleep. The scenario of drowsiness detection experiment design is summarized in Figure 3:

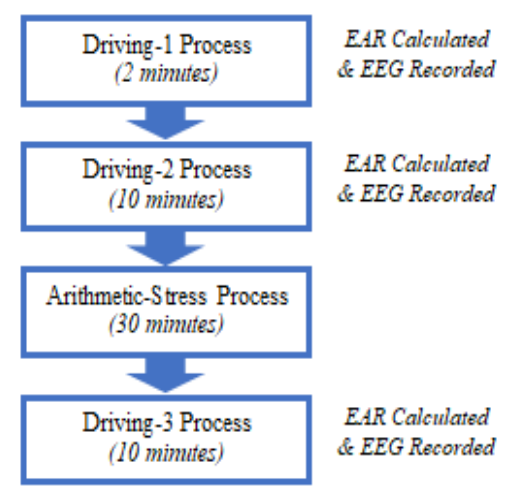

Figure 3. Scenario of drowsiness detection experiment

First stage, the entire respondent did Driving-1 process (duration: 2 minutes), this is the baseline condition of respondent. Second stage is continuing to Driving-2 process (duration: 10 minutes). Third stage is arithmetic-stress process (duration: 30 minutes), the objective of this process is to make it the respondent 
feel fatigue. The last stage is Driving-2 process (duration: 10 minutes). Total duration times of experiment are 52 minutes.

Respondent in this research using driver simulator, and the camera is placed in front of the respondent's face, so the eye of aspect area (EAR) respondent can be observed and calculated. While driving process, respondent also using the neuroheadset emotiv EPOC, to record EEG signals. Emotiv EPOC 14 channels are devices for recording electrical activity in the brain, and the location of the electrodes can be seen in Figure 4 [28].
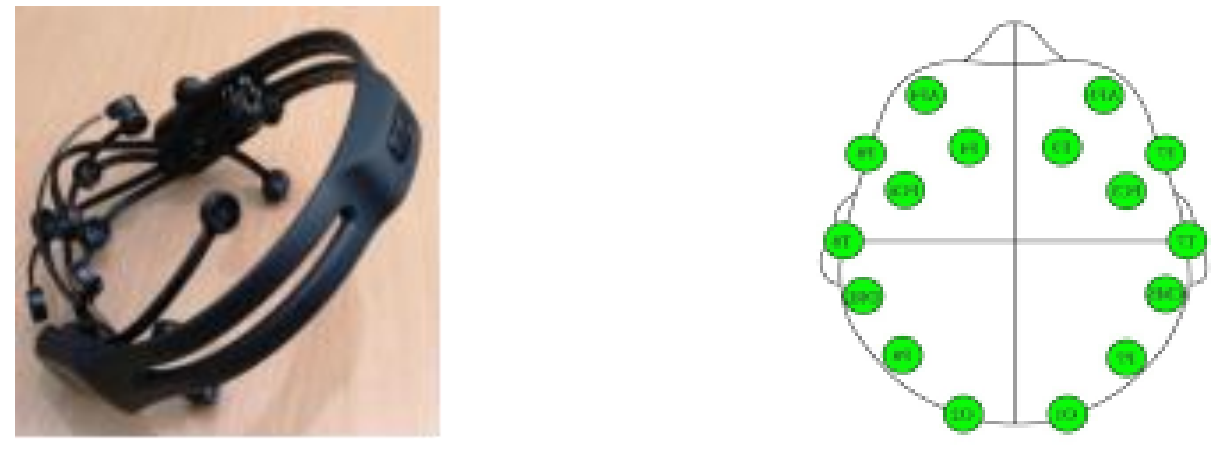

Figure 4. Neuroheadset emotiv EPOC 14 electrode channels and locations

Emotiv EPOC has several components, namely, a headset, universal USB receiver, and mini-B to A USB cable. The headset must be mounted in the right position on the respondent's head and connected to the Emotiv PRO software via a universal USB receiver. The results of this experiment are then saved. The Driving-1 process is considered a baseline condition, is the respondent's condition is considered awake. The drowsiness condition of the driver was observed based on the Eye Aspect ratio (EAR) and the respondent's EEG signal behavior from Driving-2 process and Driving-3 process. EEG signal recording is done simultaneously with EAR calculated. Block diagram of the design of drowsiness detection systems using SVM can be seen in Figure 5, which consists of several processes, namely preprocessing, feature extraction, and classification.

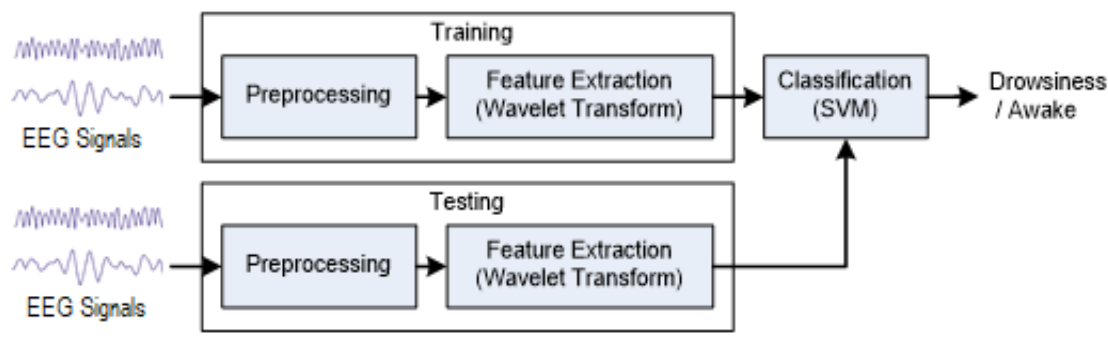

Figure 5. Drowsiness detection using wavelet transform and SVM system block diagram

\subsubsection{Preprocessing}

In this research, EEG signals were recorded using 14 channel Emotiv EPOC Neuroheadset, using frontal channels (F3, F4, F7, F8) and occipital (O1 and O2). The signals used in this research are alpha, high beta, low beta and theta. Display of EEG signal recording in Emotiv PRO software can be seen in Figure 6. At the preprocessing stage, the data of EEG signals separated to training data and testing data. For training data, EEG signals are grouped into drowsiness signals and non-drowsiness signals based on EAR. Awake condition signal was taken from the Driving-1 process, then the drowsiness condition signal was taken from Driving-2 process and Drive-3 process. If the EAR is below the threshold for 1.5 seconds or 38 consecutive frames, then the respondent is considered drowsy [6]. Then the EEG signal is segmented each 2 minutes, so from Driving-2 process and Driving-3 process there are five segmented data per 2 minutes (from 10 minutes EEG signal). 


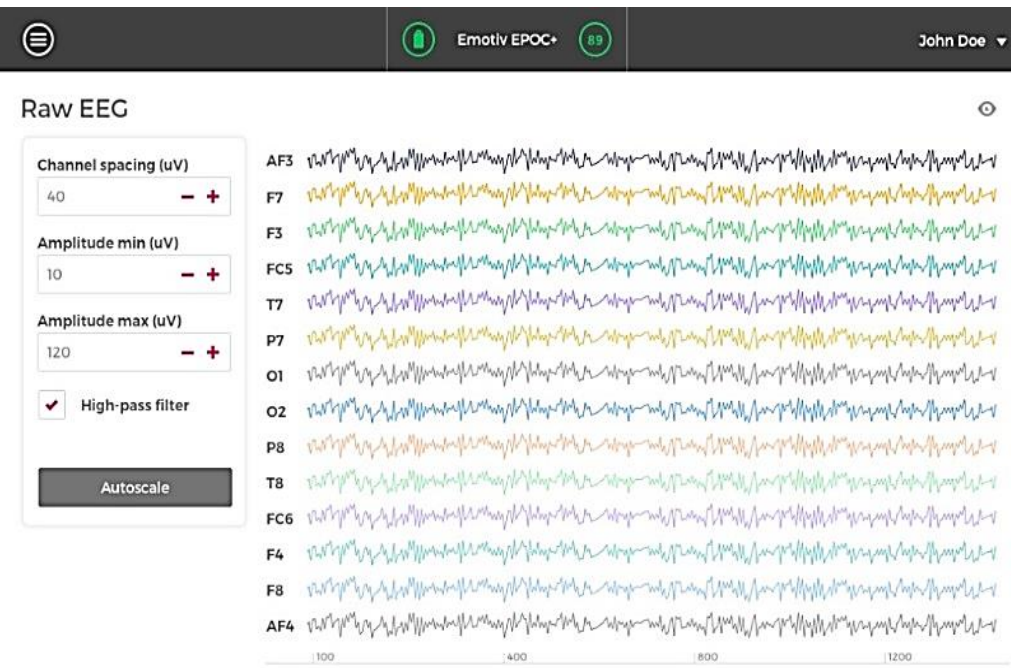

Figure 6. Display of EEG signal recording in emotiv PRO software

\subsubsection{Feature extraction}

After the signal pre-processing stage process, the next step is feature extraction. Feature extraction used in this research is wavelet transform. The wavelet type used is Daubechies (Db4) at level 4 . Level 4 was chosen because at this level the signal decomposition results most closely resemble the original signal. Figure 7(a) is the raw data signal EEG F3 Alpha, then after the wavelet transform process can be seen in Figure 7(b). Wavelet coefficients from each channel EEG signal used as feature extraction.

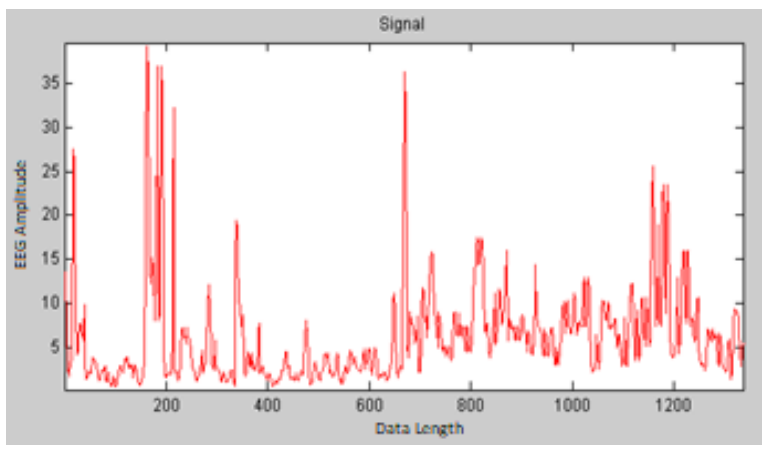

(a)

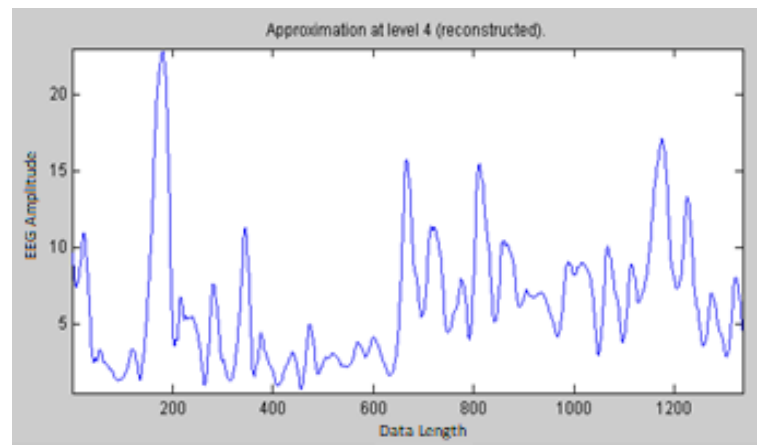

(b)

Figure 7. EEG signal of F3 alpha; (a) raw data, (b) after wavelet transform

\subsubsection{Classification}

In this research the classification method used is SVM. The validation technique used is k-fold cross validation with $\mathrm{k}=5$, chosen because for each driving process have 5 set data. In $\mathrm{K}$-fold cross-validation, dataset $X$ is randomly divided into $K$ parts of equal magnitude $X \_i, i=1,2, \ldots K$. To produce each pair of data is to store one of the $\mathrm{K}$ parts into a validation set, then combine with parts the remaining $\mathrm{K}-1$ is to form the training set. Done K times (Ethem):

$$
\begin{gathered}
V_{1}=X_{1}, T_{1}=X_{2} \cup X_{3} \cup \cdots \cup X_{k} \\
V_{2}=X_{2}, T_{2}=X_{1} \cup X_{3} \cup \cdots \cup X_{k} \\
\vdots \\
V_{k}=X_{k}, T_{k}=X_{1} \cup X_{2} \cup \cdots \cup X_{k-1}
\end{gathered}
$$

In training process, several kernel classifier SVM testing to choosen the highest accuration. Then from the best kernel chosen, continued to training process. Data classify into two classes, namely 1 
(drowsiness class) and -1 (not drowsiness/ awake class). After the SVM testing process, contains a class prediction for each data consisting of 66 outputs is the result of classification consisting of 1 (drowsiness class) and -1 (not drowsiness/ awake class). In this research, indication of drowsiness respondent is determined if at least $2 / 3$ of the 66 outputs (there are 44 output) stated "1" (drowsiness class), 2/3 (66\%) chosen because this decision greater than $50 \%$ SVM output in order to determine a clear classification for each class.

\section{RESULTS AND DISCUSSION}

Wavelet coefficients used in this research were 66 coefficients used as input to the SVM in the training and testing process. In the classification training process using SVM, the validation technique used is 5-fold cross validation. From various kernels tested, it was found that the highest level of accuracy in the SVM training process using a quadratic kernel reached $84.5 \%$. Thus the kernel used is the Quadratic kernel, see Table 1.

Table 1. Kernel accuration

\begin{tabular}{cr}
\hline Kernel & Accuration \\
\hline Linear & $71.8 \%$ \\
Quadratic & $84.5 \%$ \\
Cubic & $52.3 \%$ \\
Fine Gaussian & $72.2 \%$ \\
Medium Gaussian & $70.2 \%$ \\
Coarse Gaussian & $59.6 \%$ \\
\hline
\end{tabular}

After the training process then proceed to the testing process, which is carried out on Driving-2 process and Driving-3 process. In the Driving-2 process the results of the SVM classification of 10 respondents can be seen in Figure 8(a). If the total SVM output is greater than 2/3 from output SVM, then the class is declared as drowsiness (1), else the class is declared as awake (-1), performed on all five data for each respondent in the Driving-2 Process, see Figure 8(b). For example for respondent R1, respondent was detected drowsiness in the Data2-2, Data2-3 and Data2-4. Whereas in the Data2-1 and Data2-5 detected in the awake conditions (no drowsiness). So, totally in 10 minutes process, the driver is majority drowsy (R1, R2, R5, R6, R7, R9, and R10). And there 3 respondents were detected as awake class (R3, R4, and R8).

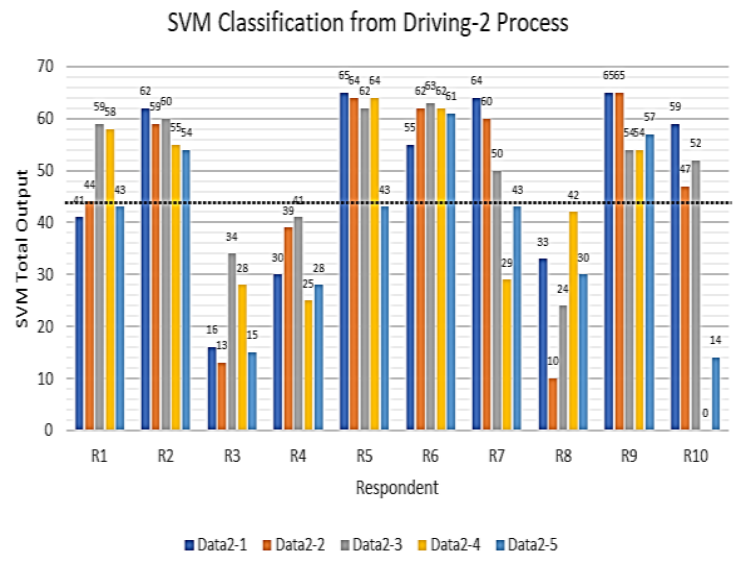

(a)

\section{Final Classification from Driving-2 Process}

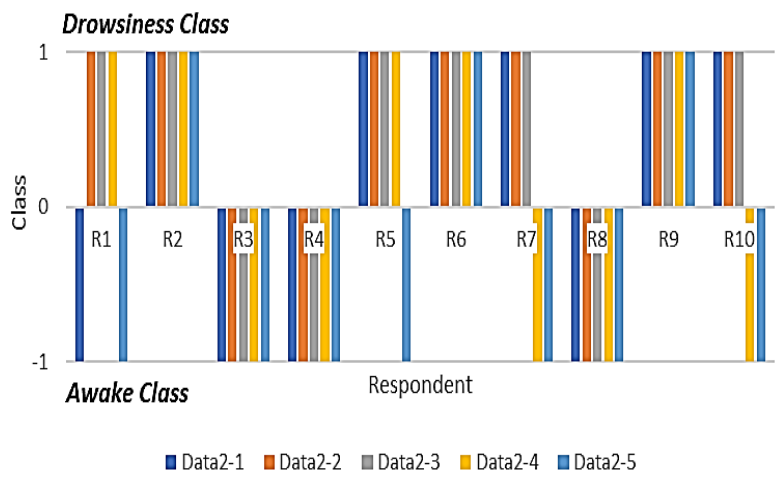

(b)

Figure 8. SVM classification and final classification from Driving-2 process; (a) SVM classification, (b) final classification

In the Drive-3 process the results of the SVM classification of 10 respondents can be seen in Figure 9(a). Then the final classification, see Figure 9(b). The final classification results can be seen that the results of the majority drowsy are respondents R1, R2, R5, R6, R7, and R9, and the respondent identified as not drowsy/ awake are respondents R3, R4, R8 and R10. 
SVM Classification from Driving-3 Process

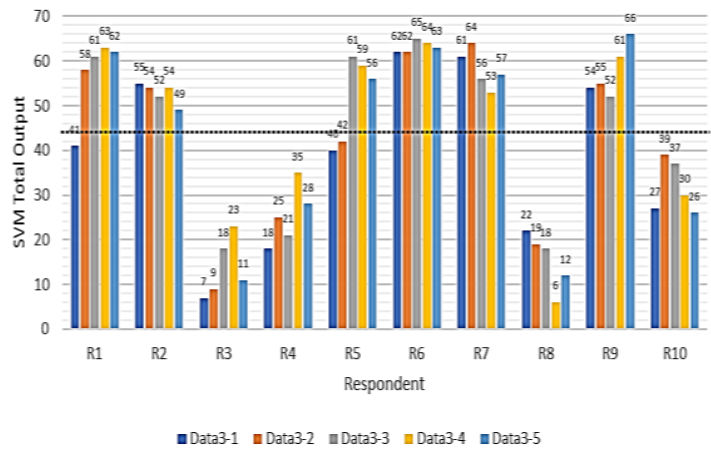

(a)
Final Classification from Driving-3 Process

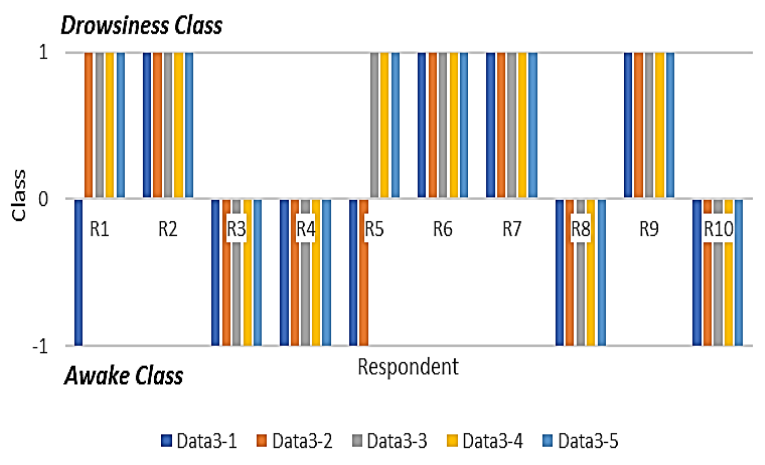

(b)

Figure 9. SVM classification and final classification from Driving-3 Process; (a) SVM classification, (b) final classification

\section{CONCLUSION}

In this research has successfully used EEG signals based on modified EAR for drowsiness detection by using wavelet transform as a feature extraction and SVM as a classifier. With 5-fold cross validation, in this research quadratic kernel $(84.5 \%)$ produces the best level of accuracy compared to the type of linear kernel $(72.2 \%)$, cubic $(52.3 \%)$, fine gaussian $(72.2 \%)$, medium gaussian $(70.2 \%)$, and coarse gaussian (59.6\%). In testing Driving-2 process data results, 7 respondents were detected as drowsiness class (R1, R2, R5, R6, R7, R9, and R10), and 3 respondents were detected as awake class (R3, R4, and R8). In Driving-3 process there 6 respondents were detected as drowsiness class (R1, R2, R5, R6, R7, and R9), and 4 respondents were detected as awake class (R3, R4, R8 and R10). Because in this experiment drowsiness detection was observed in a certain time period, so for the next research to develop drowsiness detection with a short time in real time, by utilizing several other combination methods.

\section{ACKNOWLEDGEMENTS}

Thanks to Universitas Kristen Maranatha for funding this research.

\section{REFERENCES}

[1] A. Čolić, O. Marques, and B. Furht, "Driver Drowsiness Detection," Springer International Publishing, 2014, doi: 10.1007/978-3-319-11535-1.

[2] J. G. Brown, M. Wieroney, L. Blair, S. Zhu, J. Warren, S. M. Scharf et al., "Measuring subjective sleepiness at work in hospital nurses: validation of a modified delivery format of the Karolinska Sleepiness Scale," Sleep Breath, vol. 18, no. 4, pp. 731-739, 2014, doi: 10.1007/s11325-013-0935-z.

[3] M. E. Jewett, D. J. Dijk, R. E. Kronauer, and D. F. Dinges, "Dose-response relationship between sleep duration and human psychomotor vigilance and subjective alertness," Sleep, vol. 22, no. 2, pp. 171-179, 1999, doi: 10.1093/sleep/22.2.171.

[4] N. T. Br Pasaribu, Ratnadewi, A. Prijono, R. P. Adhie, W. Halim, and R. M. Heryanto, "Performance male and female drivers in drowsiness system based on psychomotor vigilance task test," International Journal of Engineering and Technology, vol. 7, no. 2.13, pp. 421-424, 2018, doi: 10.14419/ijet.v7i2.13.16937

[5] S. Mehta, S. Dadhich, S. Gumber, and A. J. Bhatt, "Real-Time Driver Drowsiness Detection System Using Eye Aspect Ratio and Eye Closure Ratio," Proceedings of international conference on sustainable computing in science, technology and management (SUSCOM), Amity University Rajasthan, Jaipur-India, 2019, pp. 1333-1339, doi: $10.2139 /$ ssrn.3356401.

[6] N. T. Pasaribu, Ratnadewi, A. Prijono, R. P. Adhie, "Drowsiness detection system design based on individual driver," AIP Conference Proceedings, vol. 2097, no. 1, 2019, Art. no. 030104, doi: 10.1063/1.5098279.

[7] T. Vesselenyi, S. Moca, A. Rus, T. Mitran, and M. B. Tătaru, "Driver drowsiness detection using ANN image processing," IOP Conference Series Materials Science and Engineering, vol. 252, no. 1, 2017, Art. no. 012097, doi: 10.1088/1757-899X/252/1/012097.

[8] M. Ogino and Y. Mitsukura, "Portable drowsiness detection through use of a prefrontal single-channel electroencephalogram," Sensors, vol. 18, no. 12, pp. 1-19, 2018, Art. no. 4477, doi: 10.3390/s18124477.

[9] N. Gurudath and H. B. Riley, "Drowsy driving detection by EEG analysis using Wavelet Transform and K-means clustering," Procedia Computer Science, vol. 34, pp. 400-409, 2014, doi: 10.1016/j.procs.2014.07.045. 
[10] M. Awais, N. Badruddin, and M. Drieberg, "Driver drowsiness detection using EEG power spectrum analysis," 2014 IEEE Region 10 Symposium, 2014, pp. 244-247, doi: 10.1109/tenconspring.2014.6863035.

[11] M. Awais, N. Badruddin, and M. Drieberg, "A hybrid approach to detect driver drowsiness utilizing physiological signals to improve system performance and Wearability," Sensors, vol. 17, no. 9, pp. 1-16, 2017, Art. no. 1991, doi: $10.3390 / \mathrm{s} 17091991$.

[12] G. Li and W. Y. Chung, "A context-aware EEG headset system for early detection of driver drowsiness," Sensors, vol. 15, no. 8, pp. 20873-20893, 2015, doi: 10.3390/s150820873.

[13] T. Hwang, M. Kim, S. Hong, and K. S. Park, "Driver drowsiness detection using the in-ear EEG," 2016 38th Annual International Conference of the IEEE Engineering in Medicine and Biology Society (EMBC), 2016, pp. 4646-4649, doi: 10.1109/EMBC.2016.7591763.

[14] S. H. Hwang, M. Park, J. Kim, Y. Yun, and J. Son, "Driver drowsiness detection using EEG features," International Conference on Human-Computer Interaction- HCI 2018, vol. 852, 2018, pp. 367-374, doi: 10.1007/978-3-319-92285-0_49.

[15] Korlantas, "korlantas.” 2018, [Online]. Available: http://korlantas-irsms.info/graph/accidentData

[16] J. N. Mindoro, C. D. Casuat, A. S. Alon, M. A. F. Malbog, and J. A. B. Susa, "Drowsy or not?Early drowsiness detection utilizing arduino based on electroencephalogram (eeg) neuro-signal," International Journal of Advanced Trends in Computer Science and Engineering, vol. 9, no. 2, pp. 2221-2226, 2020, doi: 10.30534/ijatcse/2020/200922020.

[17] M. Akin, M. B. Kurt, N. Sezgin, and M. Bayram, "Estimating vigilance level by using EEG and EMG signals," Neural Computing and Applications, vol. 17, no. 3, pp. 227-236, 2008, doi: 10.1007/s00521-007-0117-7.

[18] G. Li and W. Y. Chung, "Detection of Driver Drowsiness using Wavelet Analysis of Heart Rate Variability and a Support Vector Machine Classifier," Sensors, vol. 13, no. 12, pp. 16494-16511, 2013, doi: 10.3390/s131216494.

[19] Y. Zhang, B. Liu, X. Ji, and D. Huang, "Classification of EEG Signals Based on Autoregressive Model and Wavelet Packet Decomposition," Neural Processing Letters, vol. 45, no. 2, pp. 365-378, 2017, doi: 10.1007/s11063-016-9530-1.

[20] L. Pauly and D. Sankar, "Detection of drowsiness based on HOG features and SVM Classifiers," 2015 IEEE International Conference on Research in Computational Intelligence and Communication Networks (ICRCICN), 2015, pp. 181-186, doi: 10.1109/ICRCICN.2015.7434232.

[21] X. Zhang, J. Li, Y. Liu, Z. Zhang, Z. Wang, D. Luo et al., "Design of a fatigue detection system for high-speed trains based on driver vigilance using a wireless wearable EEG," Sensors, vol. 17, no. 3, 2017, Art. no. 486, doi: $10.3390 / \mathrm{s} 17030486$.

[22] N. Kasabov, "Springer handbook of bio-/neuroinformatics," Berlin, Heidelberg: Springer Berlin Heidelberg, 2014, doi: 10.1007/978-3-642-30574-0.

[23] R. Polikar, "The Wavelet Tutorial," $2006 . \quad$ [Online]. Available: http:://users.rowan.edu/ polikar/WAVELETS/WTtutorial.html.

[24] K. P. Soman, N. G. Resmi, and K. I. Ramachandran, "Insight Into Wavelets: From Theory to Practice," PHI Learning Pvt. Ltd., 2010.

[25] C. Cortes and V. Vapnik, "Support-vector networks," Machine Language, vol. 20, no. 3, pp. 273-297, 1995, doi: 10.1007/BF00994018.

[26] M. N. Murty and R. Raghava, "Support Vector Machines and Perceptrons," Springer International Publishing, 2016, doi: 10.1007/978-3-319-41063-0.

[27] A. Statnikov, C. F. Aliferis, D. P. Hardin, and I. Guyon, "A Gentle Introduction to Support Vector Machines in Biomedicine," World Scientific, 2011, doi: 10.1142/7922

[28] F. E.-Z. Fouad, F. El-Zahraa Mohammaed, "Using Emotiv EPOC Neuroheadset To Acquire Data In BrainComputer Interface," International Journal of Advanced Research, vol. 3, no. 11, pp. 1012-1017, 2016. 